\title{
The Association of Defects and Zinc in Hematite
}

\section{Supporting Information}

Eric J. Bylaska ${ }^{*}$, Jeffrey G. Catalano ${ }^{2}$, Sebastian T. Mergelsburg ${ }^{1}$, Sarah A. Saslow ${ }^{1}$, Odeta Qafoku $^{1}$, Micah P. Prange ${ }^{1}$, Eugene S. Ilton ${ }^{1 *}$

${ }^{1}$ Pacific Northwest National Laboratory, Richland WA, 99352, USA

${ }^{2}$ Department of Earth and Planetary Sciences, Washington University, St. Louis, MO 63130, USA

*Corresponding Authors: Eugene.Ilton@pnnl.gov; Eric.Bylaska@pnnl.gov 


\section{Preparation of ZnHem_FC}

Sample ZnHem_FC was originally prepared and characterized by Frierdich et al. (2012). The synthesis procedure used was adapted from previously reported methods (Schwertmann and Cornell, 2000). Zn-substituted hematite was prepared by slow addition of $600 \mathrm{~mL}$ of a solution containing $0.196 \mathrm{M}$ iron(III) nitrate nonahydrate and $0.004 \mathrm{M}$ zinc chloride to $360 \mathrm{~mL}$ of $1 \mathrm{M}$ sodium hydroxide contained in a polypropylene bottle. All chemicals used were ACS grade or better and obtained from either Sigma-Aldrich or Fisher Scientific and solutions were prepared in polypropylene volumetric flasks to prevent silica contamination of the surface. The $\mathrm{pH}$ of the resulting suspension was adjusted by 8.5 by dropwise addition of 0.1 or $1.0 \mathrm{M}$ sodium hydroxide. This suspension was then capped and heated in a convection oven with a digital temperature controller at $98^{\circ} \mathrm{C}$ for 11 days. After this aging step the suspension was removed from the oven and cooled to room temperature. The suspension was divided up into $250 \mathrm{~mL}$ polypropylene bottles, centrifuged at $4500 \mathrm{rpm}$ until the particle settled using a Beckman Coulter Allegra X-22R centrifuge and a SX4250 swinging bucket rotor. The synthesis fluid was decanted and the Znsubstituted hematite was then suspended in $1 \mathrm{M}$ hydrochloric acid for $2 \mathrm{~h}$ to remove residual adsorbed Zn from the mineral surface. The suspension was then repeatedly centrifuged, decanted, and resuspended in deionized water $(>18.2 \mathrm{M} \Omega \cdot \mathrm{cm})$ to wash it free of electrolytes. This resulted in a $250 \mathrm{~mL}$ suspension of $\mathrm{Zn}$-substituted hematite in deionized water, which was used for the experiments described in Frierdich et al. (2012). A subsample of this final suspension was removed and dried overnight in an oven at $70^{\circ} \mathrm{C}$ to obtain powder that was used for sample characterization, as reported in Frierdich et al. (2012). An archived sample of this dried Zn-substituted hematite is employed in the present study as sample ZnHem_FC. 


\section{X-ray diffraction patterns and analysis}

Powder XRD analysis were performed using a Philips X'Pert MPD system with a vertical goniometer. The X-ray source is a long-fine-focus ceramic X-ray tube with a Cu anode. Data were collected at a $2 \theta$ range of 2 to $75^{\circ}$ with a scan step of $0.005^{\circ}$, acquisition of 4 seconds/step, acceleration voltage of $50 \mathrm{kV}$, and beam current of $40 \mathrm{~mA}$.

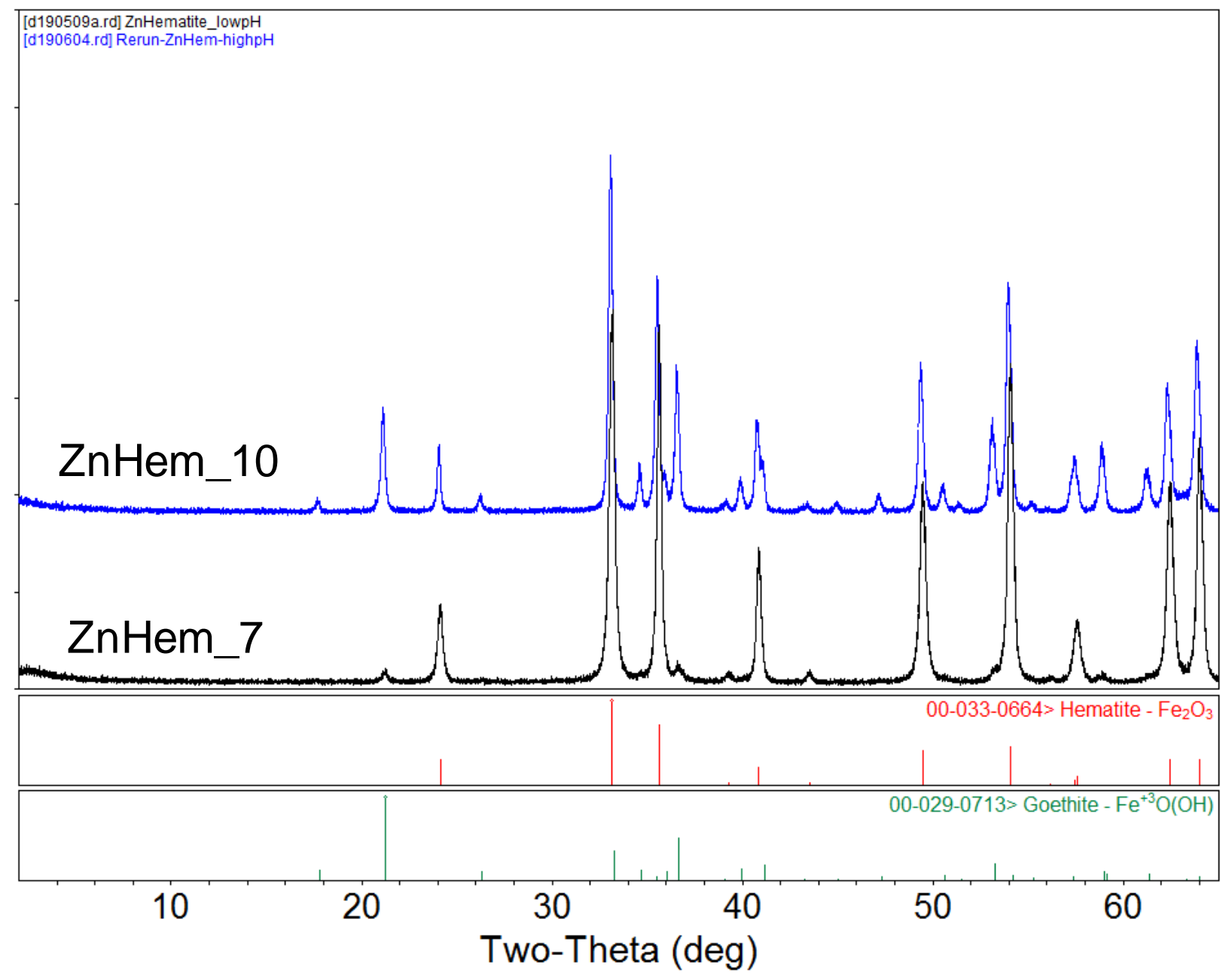

Figure S1: Comparison of XRD for samples ZnHem_10 and ZnHem_7 


\begin{tabular}{lcccccccccc}
\hline sample & wt\% hem & wt \% goe & $\mathrm{R}_{\mathrm{w}}(\%)$ & $\mathrm{R}_{\exp }(\%)$ & $\chi^{2}$ & $a_{\text {hem }}$ & $C$ hem & $a_{\text {goe }}$ & $b_{\text {goe }}$ & $c_{\text {goe }}$ \\
\hline ZnHem_7 & 96.3 & 3.7 & 25.48 & 16.45 & 2.40 & 5.0343 & 13.7480 & 4.5924 & 9.9961 & 3.0175 \\
ZnHem_10 & 61.6 & 38.4 & 24.61 & 17.62 & 1.95 & 5.0451 & 13.7763 & 4.6177 & 9.9847 & 3.0271 \\
\hline
\end{tabular}
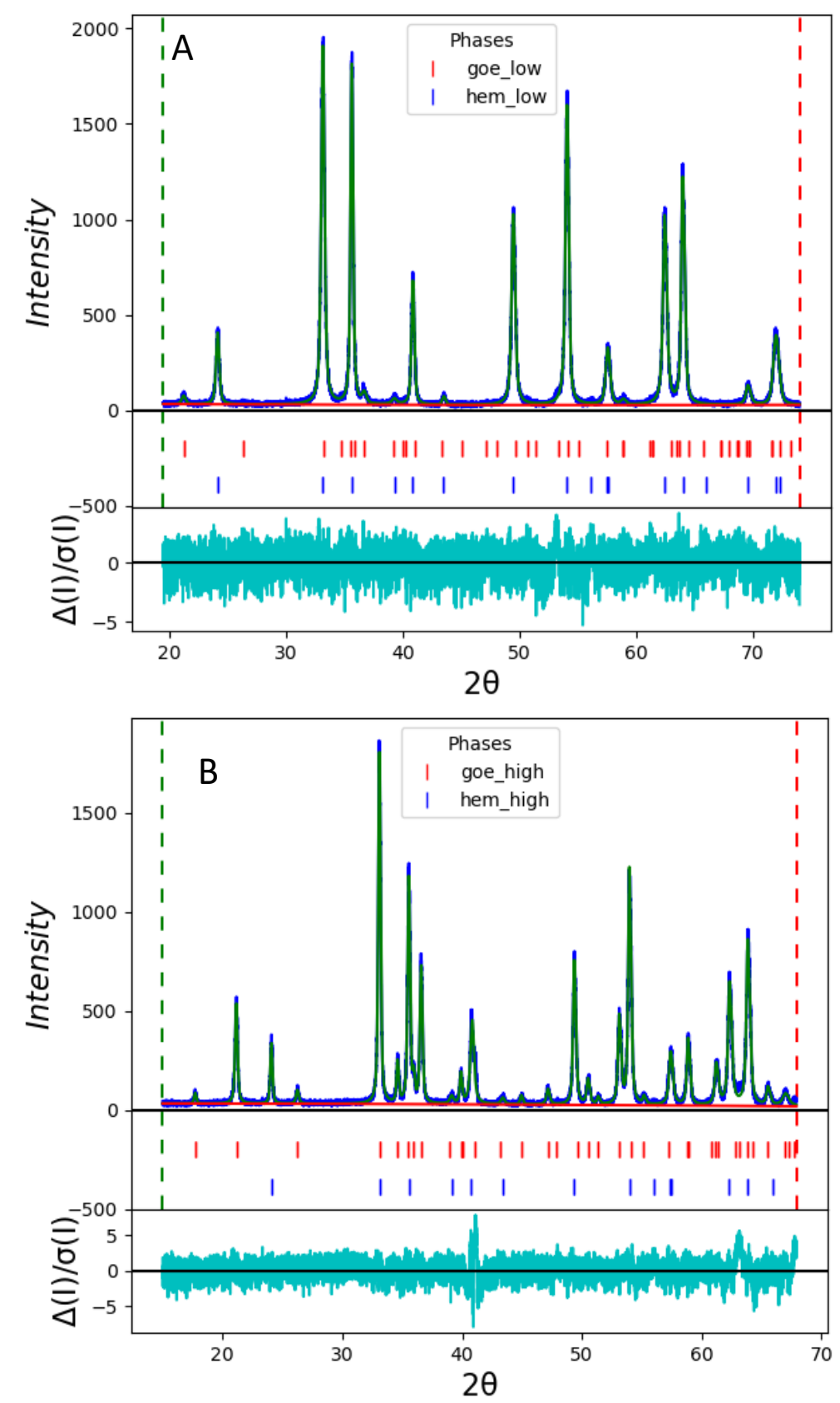

Fig S2. Refinement of ZnHem_7 (A) and ZnHem_10 (B) XRD patterns using calculated reference structures of goethite (red) and hematite (blue). The original data set is shown as the blue line, the calculated fit is green, and the residual is plotted below in teal. 


\section{Scanning electron microscopy}

Scanning Electron Microscopy (SEM) analyses were conducted with a FEI Helios NanoLab 600i field emission electron microscope. Prior to analysis, a small amount of powders were mounted on a SEM aluminum stub and were coated with a $10 \mathrm{~nm}$ carbon layer by thermal evaporation using a 108C Auto Carbon Coater (Ted Pella, Inc.). Particle morphology was examined using a secondary electron Everhart-Thornley detector (ETD) in a field free mode at an acceleration voltage of 3 to $5 \mathrm{kV}$ and a current of 0.086 to $0.17 \mathrm{nA}$. Higher magnification images were collected in immersion mode with through-lens-detector (TLD) using same voltage/current as described above.

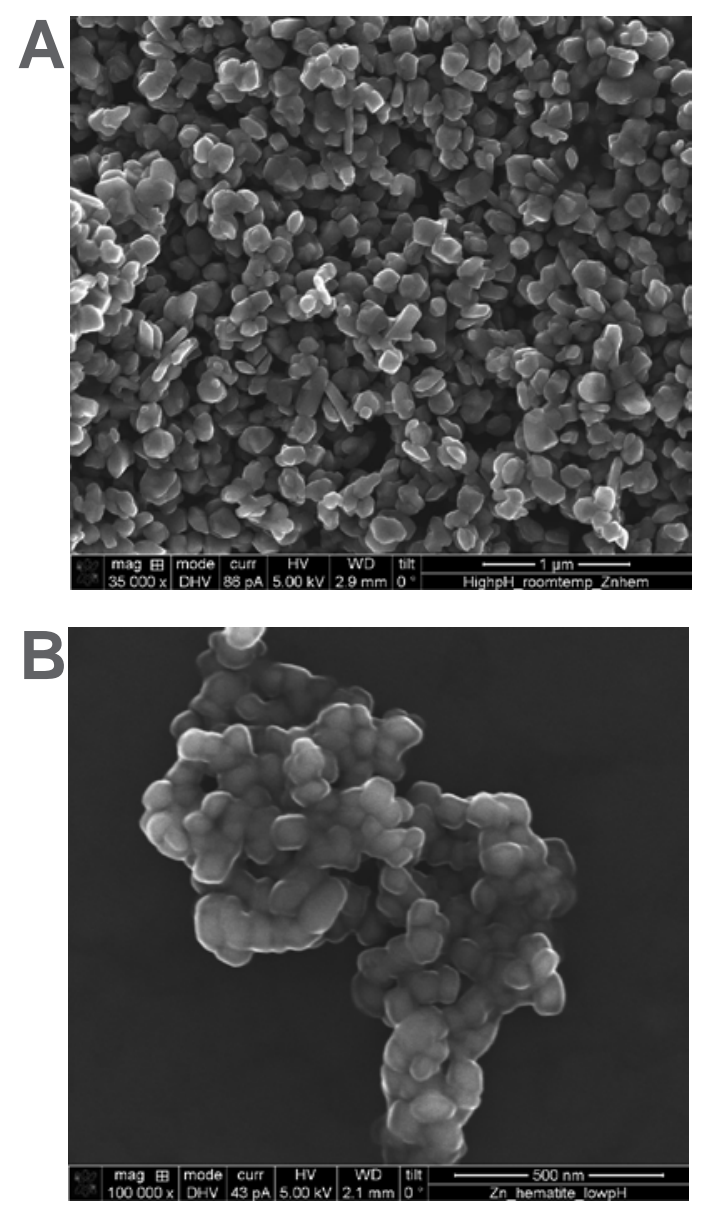

Figure S3. SEM of (A) ZnHem_10 and (B) ZnHem_7. Goethite visible in (A) are lath-like, not needles. 


\section{Extended X-ray absorption fine structure (EXAFS)}

EXAFS sample preparation and analysis of ZnHem_7 and ZnHem_10 were performed as follows: $~ 100 \mathrm{mg}$ from the respective solid was homogeneously packed into a Teflon window with Kapton tape on one side and sealed with a second piece of Kapton tape after sample packing. Data for the water rinsed samples were obtained at the Stanford Synchrotron Radiation Lightsource (SSRL) beamline 11-2 at the Zn K edge (9659 eV) using a double crystal monochromator with Si (220), $\varphi=90^{\circ}$ orientation crystals detuned by 50\%. Data were collected from $200 \mathrm{eV}$ below the edge to $\sim 900 \mathrm{eV}$ above the edge $\left(\mathrm{k}=15 \AA^{-1}\right)$. Data between $200 \mathrm{eV}$ to $20 \mathrm{eV}$ below the edge were collected with $10 \mathrm{eV}$ spacing and from $20 \mathrm{eV}$ below the edge to $30 \mathrm{eV}$ above the edge data were collected with $0.35 \mathrm{eV}$ spacing. The remaining data above the edge was obtained with a k-spacing of $0.07 \AA^{-1}$ and the collection time was weighted by $\mathrm{k}^{3}$. Reference spectra (Zn metal foil) were collected in transmission mode using $\mathrm{N}_{2}$ filled ion chambers. Fluorescence data were obtained using a 100 element Ge detector and were corrected for detector dead time. Data were reduced from raw data to spectra using SixPack ${ }^{1}$. Zn K edge data for the acid washed samples were collected at the Advanced Photon Source (APS) beamline 20-BM using a double crystal monochromator with Si (111) crystals detuned by 15\%. Data were collected from $200 \mathrm{eV}$ below the edge to $\sim 970 \mathrm{eV}$ above the edge $\left(\mathrm{k}=16 \AA^{-1}\right)$. Data between $200 \mathrm{eV}$ to $20 \mathrm{eV}$ below the edge were collected with $10 \mathrm{eV}$ spacing and from $20 \mathrm{eV}$ below the edge to $50 \mathrm{eV}$ above the edge data were collected with $0.35 \mathrm{eV}$ spacing. The remaining data above the edge was obtained with a kspacing of $0.05 \AA^{-1}$ and the collection time was weighted by $\mathrm{k}^{3}$. Reference spectra were collected in transmission mode using $\mathrm{N}_{2}$ filled ion chambers (post-sample ion chambers only, Iт and IrEF). The ion chamber before the sample ( $\left.\mathrm{I}_{0}\right)$ was filled with $50 \% \mathrm{~N}_{2}$ and $50 \%$ He. Fluorescence data 
were obtained using a 13 element Ge detector. Spectra from both the APS and SSRL were normalized and energy calibrated using Zn metal foil as the energy reference in Athena ${ }^{2}$.

\section{Ab initio molecular dynamic simulations of $\mathrm{Zn} \mathrm{K}$-edge for $\mathrm{Zn}$ incorporated in hematite}

All DFT optimization and Car-Parrinello simulations ${ }^{3,4}$ in this study were performed with the

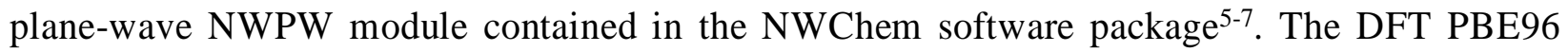
exchange correlation function ${ }^{8}$ was used for these calculations. The interactions between valence electrons and the atom centers were approximated using generalized norm-conserving Hamann pseudopotentials $^{9,10}$ for $\mathrm{O}$, and $\mathrm{H}$ and a norm-conserving Troullier-Martin pseudopotential ${ }^{11}$, which contained $4 s, 4 p$, and $3 d$ projectors and a semi-core correction was used for Fe. The pseudopotentials were modified into a separable form as suggested by Kleinman and Bylander ${ }^{12}$. For gradient corrected calculations, the NWPW module automatically generates pseudopotentials using the specified exchange correlation functional. The original pseudopotential parameterization suggested by Hamann were slightly softened by increasing the core radii: H: $\mathrm{r}_{\mathrm{cs}}=0.8$ a.u., $\mathrm{r}_{\mathrm{cp}}=0.8$ a.u.; O: $\mathrm{r}_{\mathrm{cs}}=0.7$ a.u., $\mathrm{r}_{\mathrm{cp}}=0.7$ a.u., and $\mathrm{r}_{\mathrm{cd}}=0.7$ a.u. The radial cutoffs for the Troullier-Martin pseudopotential for $\mathrm{Fe}$ were $\mathrm{r}_{\mathrm{cs}}=1.24$ a.u., $\mathrm{r}_{\mathrm{cp}}=1.24$ a.u., $\mathrm{r}_{\mathrm{cd}}=1.23$ a.u., and the s-channel pseudopotentials was chosen for the local potential in the Kleinman and Bylander expansion ${ }^{13}$. Since this is a spin ordered system, unrestricted DFT calculations were performed. The electronic wavefunctions were expanded using a plane-wave basis with periodic boundary conditions at the $\Gamma$-point with a wavefunction cutoff energy of 100 Ry and a density cutoff energy of $200 \mathrm{Ry}$. The host crystal structure used for these simulations was a $2 \times 2 \times 2$ rhombohedral hematite super-cell $\left(\mathrm{Fe}_{32} \mathrm{O} 48 ; \mathrm{a}=\mathrm{b}=\mathrm{c}=10.99 \AA ; \alpha=\beta=\gamma=55.2^{\circ}\right)$. The lattice constants used for this host supercell $\left(\mathrm{a}=\mathrm{b}=\mathrm{c}=10.99 \AA ; \alpha=\beta=\gamma=55.2^{\circ}\right)$ were obtained by optimizing the 72-atom unit cell with 
open shell DFT PBE96 $\Gamma$-point calculations. This lattice structure was used for all the defected Zn containing hematite structures and a spin-penalty scheme was used to initialize the antiferromagnetic configurations.

AIMD simulations were performed for at least 20 ps for the 21 independent configurations in which a $\mathrm{Zn}$ ion was incorporated in the hematite structure. In these simulations, the system was propagated in time using Car-Parrinello molecular dynamics (CPMD) scheme. The same exchange-correlation potential, pseudopotential and cutoff energies used in plane-wave optimization part were used in the dynamic AIMD simulations. Equation of motions in CPMD were integrated using position Verlet algorithm, with a time step 0.12 fs and fictitious orbital mass $750.0 \mathrm{au}$. All hydrogen atoms were replaced by deuterium to facilitate the integration. The simulation was carried out in a constant temperature canonical ensemble (300 K) using NoseHoover thermostats to control the temperatures of the ions and the 1-electron orbitals ${ }^{14-16}$. 

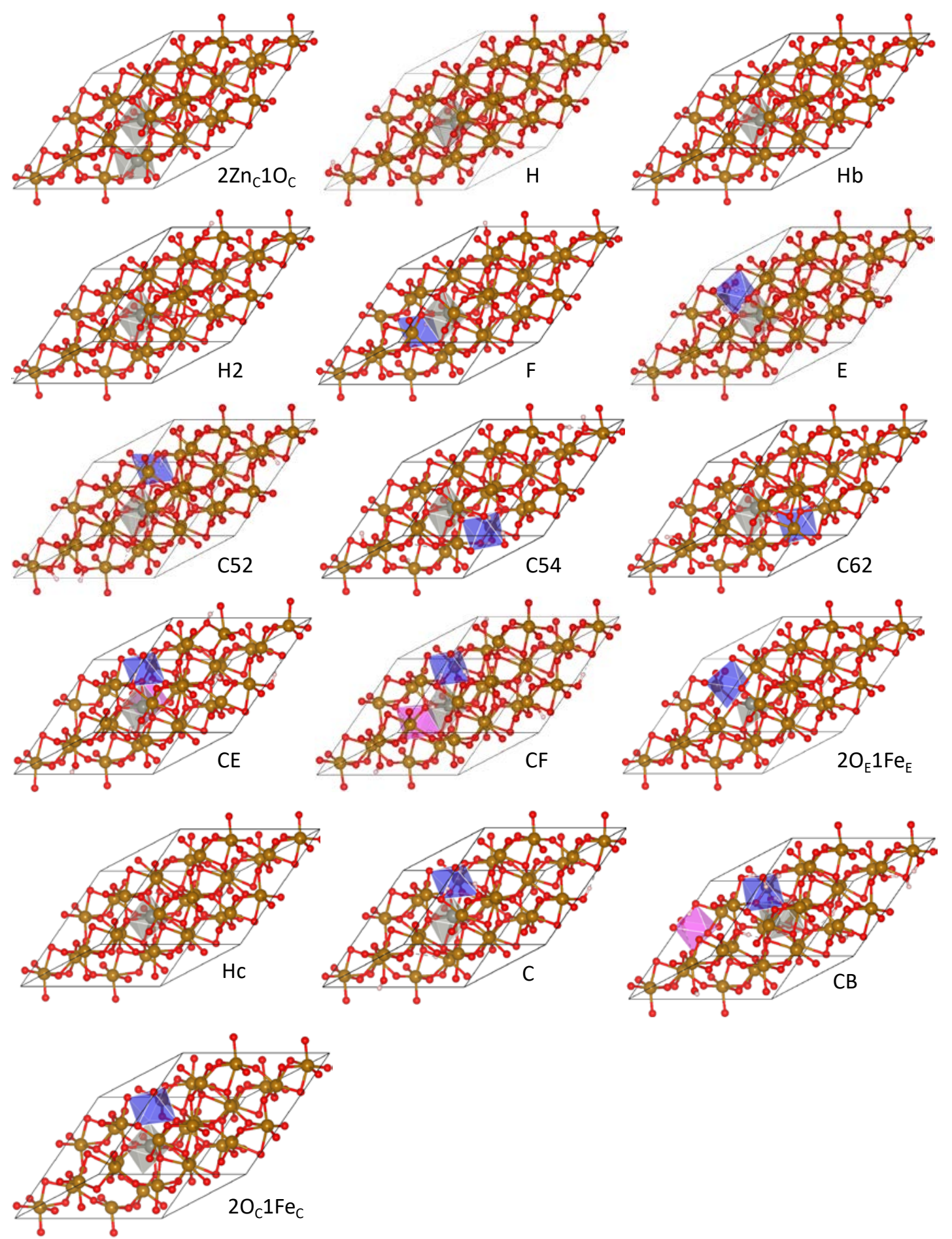

Figure S4: Molecular models for all AIMD simulated configurations of Zn incorporated in hematite. Red, orange and white balls mark oxygen, iron, and protons, respectively. Grey octahedra represent $\mathrm{Zn}$ sites and blue octahedra are protonated Fe vacancies. $\mathrm{C}, \mathrm{F}$, and $\mathrm{E}$ indicate protonated Fe vacancies that are corner, face, and edge sharing with $\mathrm{Zn}$ octahedra. B is a bulk protonated Fe vacancy. $\mathrm{H}$ and $\mathrm{H} 2$ do not have protonated Fe vacancies, but do have 1 and 2 protons present, respectively. Please refer to the caption to Figure $\mathrm{S} 5$ for more details concerning the labeling. 


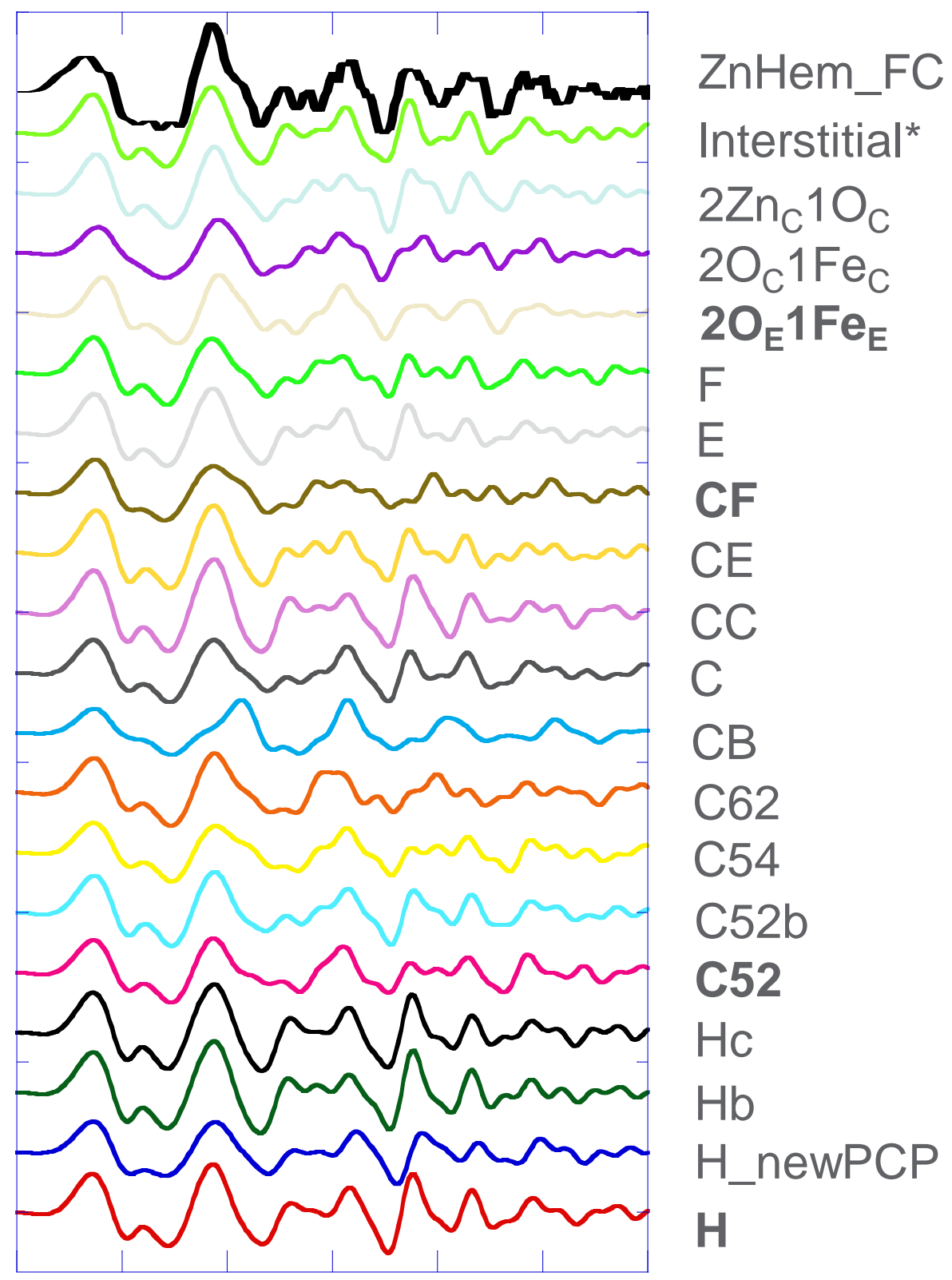

Figure S5. Simulated Zn K-edge EXAFS for all AIMD calculated Zn configurations in hematite. All configurations not involving an $\mathrm{O}$ vacancy have 1 proton (the $\mathrm{CCP}$ )in an interstitial site for charge compensation. $\mathrm{C}, \mathrm{F}$, and $\mathrm{E}$ indicate protonated Fe vacancies that are corner, face, and edge sharing with Zn octahedra. B is a bulk protonated Fe vacancy. Numbers after $\mathrm{C}$ indicate unique corner sharing sites (see Figure S4). H does not have protonated Fe vacancies. H_new PCP is the same as $\mathrm{H}$ except that a new potential was tested for Fe in the AIMD calculations. Not shown are configurations $\mathrm{H}$ simulated with a larger cell (similar to results with smaller cell) and H2 (two CCPs and a +1 charged cell). Lower case letters indicate different interstitial sites for the CCP. $2 \mathrm{Zn}_{\mathrm{c}} 1 \mathrm{O}_{\mathrm{c}}$ is $2 \mathrm{Zn}$ substituting fpr 2 corner sharing Fe with the shared $\mathrm{O}$ eliminated. 2Oc1Fec is $\mathrm{Zn}$ substituted for $1 \mathrm{Fe}$ with 2 and 1 trans-corner $\mathrm{O}$ and Fe vacancies, respectively. 2OE1FeE is $1 \mathrm{Zn}$ substituted for Fe with 2 and 1 edge sharing $\mathrm{O}$ and Fe vacancies, respectively. Bolded configurations have weight in lcf's fits to the experimental hematite data. 


\section{Table S1}

Average $\mathrm{Zn}-\mathrm{O}$ distances in each model and reference structure. Each row represents equivalent oxygen atoms; highlighted values correspond to oxygen atoms within bonding distance

\begin{tabular}{|c|c|c|c|c|c|c|c|c|c|c|c|c|c|c|c|c|c|c|c|c|c|}
\hline & Hem1* & $\mathrm{Hem}{ }^{+}$ & $\mathrm{Zn}$ & $\mathrm{H}$ & $\mathrm{Hb}$ & $\mathrm{Hc}$ & $\mathrm{H} 2$ & $\mathrm{C}$ & $\mathrm{C52}$ & C54 & C62 & $E$ & $\mathrm{~F}$ & $\mathrm{CB}$ & CC & $\mathrm{CE}$ & $\mathrm{CF}$ & $2 \mathrm{O}_{\mathrm{E}} 1 \mathrm{Fe}_{\mathrm{E}}$ & $2 \mathrm{Zn}_{\mathrm{C}} 1 \mathrm{O}$ & $2 \mathrm{Zn}_{\mathrm{C}} 1 \mathrm{O}$ & $2 \mathrm{O}_{\mathrm{C}} 1 \mathrm{Fe}$ \\
\hline M-O1 & 2.116 & 2.114 & 2.192 & 2.235 & 2.178 & 2.258 & 2.214 & 2.194 & 2.285 & 2.202 & 2.321 & 2.143 & 2.263 & 2.954 & 2.159 & 2.213 & 2.199 & 2.086 & & & $\frac{c}{2.497}$ \\
\hline M-O2 & 2.116 & 2.114 & 2.230 & 2.243 & 2.181 & 2.163 & 2.255 & 2.451 & 2.421 & 2.519 & 2.473 & 2.238 & 2.286 & 2.974 & 2.189 & 2.183 & 2.168 & 2.190 & 2.205 & 2.166 & 2.164 \\
\hline M-O3 & 2.116 & 2.114 & 2.237 & 2.194 & 2.246 & 2.192 & 2.296 & 2.219 & 2.161 & 2.241 & 2.102 & 2.246 & 2.198 & 2.835 & 2.191 & 2.216 & 2.393 & & 2.166 & 2.166 & 2.158 \\
\hline M-O4 & 1.946 & 1.944 & 2.035 & 1.981 & 2.021 & 1.979 & 1.957 & 1.989 & 1.928 & 1.930 & 1.981 & 2.007 & 1.961 & 2.868 & 1.982 & 1.990 & 2.024 & & 2.015 & 2.111 & \\
\hline M-O5 & 1.946 & 1.944 & 1.999 & 2.000 & 1.833 & 2.002 & 2.010 & 1.986 & 1.978 & 1.977 & 2.013 & 2.013 & 2.010 & 2.708 & 2.009 & 2.032 & 1.860 & 1.967 & 1.968 & 1.993 & 2.027 \\
\hline M-O6 & 1.946 & 1.944 & 2.007 & 1.993 & 2.019 & $\begin{array}{l}1.992 \\
\text {. }\end{array}$ & 1.905 & 1.887 & 1.986 & 1.969 & 1.955 & 2.019 & 1.991 & $\begin{array}{l}1.802 \\
\text {. }\end{array}$ & 1.937 & 1.953 & 1.847 & 2.003 & 1.986 & 1.977 & 1.953 \\
\hline M-O $7 *$ & & & & & & & & & & & & & & 1.841 & & & & & & & \\
\hline M-O8* & & & & & & & & & & & & & & 1.950 & & & & & & & \\
\hline$\langle\mathrm{M}-\mathrm{O}\rangle$ & 2.031 & 2.029 & 2.117 & 2.108 & 2.080 & 2.097 & 2.106 & 2.121 & 2.126 & 2.140 & 2.141 & 2.111 & 2.118 & 2.492 & 2.078 & 2.098 & 2.082 & 2.057 & 2.068 & 2.083 & 2.160 \\
\hline
\end{tabular}

* Maslen, E. N. (1994). Streltsov. VA, Streltsova NR, Ishizawa N., Hematite. Acta Crystallogr. B, 50, $435-441$.

${ }^{\dagger}$ Blake, R. L., Hessevick, R. E., Zoltai, T., \& Finger, L. W. (1966). Refinement of the hematite structure. American Mineralogist: Journal of Earth and Planetary Materials, 51(1-2), 123-129.

Table S2. Variability in proton positions for all configurations that include protons and have weight in the lcfs.

\begin{tabular}{lllllll}
\hline model & atom & $<\mathrm{O}-\mathrm{H}>(\AA)$ & $\mathrm{U}_{\text {iso }}\left(\AA^{2}\right)$ & position & $\mathrm{N}(\mathrm{O})^{*}$ & $\tau(\%)^{\dagger}$ \\
\hline $\mathrm{H}$ & $\mathrm{H}_{1}$ & 0.971 & 0.085 & interstitial & 1 & $100 \%$ \\
\hline $\mathrm{C} 52$ & $\mathrm{H}_{1}$ & 0.982 & 0.127 & Fe vacancy & 1 & $98.8 \%$ \\
& $\mathrm{H}_{2}$ & 0.959 & 0.090 & Fe vacancy & 1 & $100 \%$ \\
& $\mathrm{H}_{3}$ & 0.960 & 0.201 & interstitial & 1 & $100 \%$ \\
& $\mathrm{H}_{4}$ & 0.971 & 0.041 & Fe vacancy & 1 & $99.8 \%$ \\
\hline $\mathrm{E}$ & $\mathrm{H}_{1}$ & 0.973 & 0.039 & Fe vacancy & 1 & $100 \%$ \\
& $\mathrm{H}_{2}$ & 0.976 & 0.037 & Fe vacancy & 1 & $99.9 \%$ \\
& $\mathrm{H}_{3}$ & 0.962 & 0.060 & Fe vacancy & 1 & $100 \%$ \\
& $\mathrm{H}_{4}$ & $1.031,1.051$ & 0.133 & interstitial & 2 & $73.4 \%, 24.8 \%$ \\
\hline $\mathrm{CF}$ & $\mathrm{H}_{1}$ & 0.981 & 0.037 & Fe vacancy & 1 & $99.3 \%$ \\
& $\mathrm{H}_{2}$ & 0.959 & 0.110 & Fe vacancy & 1 & $100 \%$ \\
& $\mathrm{H}_{3}$ & 0.966 & 0.068 & Fe vacancy & 1 & $100 \%$ \\
& $\mathrm{H}_{4}$ & $1.001,1.097$ & 0.493 & interstitial & 2 & $55.9 \%, 41.4 \%$ \\
& $\mathrm{H}_{5}$ & 0.963 & 0.094 & Fe vacancy & 1 & $100 \%$ \\
& $\mathrm{H}_{6}$ & 0.961 & 0.081 & Fe vacancy & 1 & $100 \%$ \\
& $\mathrm{H}_{7}$ & 0.961 & 0.094 & Fe vacancy & 1 & $100 \%$ \\
\hline
\end{tabular}

*number of oxygen atoms within bonding distance $(<1.05 \AA){ }^{\dagger}$ Percent of frames the $\mathrm{O}-\mathrm{H}$ distance is $<1.05 \AA$.

\section{References}

1. Webb, S. M., SIXPack: a Graphical User Interface for XAS Analysis Using IFEFFIT. Physica Scripta 2005, T115, 1011-1014.

2. Ravel, B.; Newville, M., ATHENA, ARTEMIS, HEPHAESTUS: data analysis for X-ray absorption spectroscopy using IFEFFIT. Journal of Synchrotron Radiation 2005, 12, 537-541.

3. Car, R.; Parrinello, M., UNIFIED APPROACH FOR MOLECULAR-DYNAMICS AND DENSITYFUNCTIONAL THEORY. Physical Review Letters 1985, 55, (22), 2471-2474.

4. Marx, D.; Hutter, J., Ab initio molecular dynamics: basic theory and advanced methods. Cambridge University Press: Cambridge, 2012. 
5. Bylaska, E. J.; Tsemekhman, K.; Govind, N.; Valiev, M., Large-scale plane-wave-based densityfunctional theory: formalism, parallelization, and applications. In Computational Methods for Large Systems:

Electronic Structure Approaches for Biotechnology and Nanotechnology, Reimers, J. R., Ed. Wiley: 2011; pp 77-116.

6. Valiev, M.; Bylaska, E. J.; Govind, N.; Kowalski, K.; Straatsma, T. P.; Van Dam, H. J. J.; Wang, D.; Nieplocha, J.; Apra, E.; Windus, T. L.; de Jong, W., NWChem: A comprehensive and scalable open-source solution for large scale molecular simulations. Computer Physics Communications 2010, 181, (9), 14771489.

7. Bylaska, E. J., Plane-Wave DFT Methods for Chemistry. In Annual Reports in Computational Chemistry, Elsevier: 2017; Vol. 13, pp 185-228.

8. Perdew, J. P.; Burke, K.; Ernzerhof, M., Generalized gradient approximation made simple (vol 77, pg 3865, 1996). Physical Review Letters 1997, 78, (7), 1396-1396.

9. Hamann, D. R., GENERALIZED NORM-CONSERVING PSEUDOPOTENTIALS. Physical Review $B$ 1989, 40, (5), 2980-2987.

10. Hamann, D. R.; Schluter, M.; Chiang, C., NORM-CONSERVING PSEUDOPOTENTIALS. Physical Review Letters 1979, 43, (20), 1494-1497.

11. Troullier, N.; Martins, J. L., EFFICIENT PSEUDOPOTENTIALS FOR PLANE-WAVE CALCULATIONS. Physical Review B 1991, 43, (3), 1993-2006.

12. Kleinman, L.; Bylander, D. M., EFFICACIOUS FORM FOR MODEL PSEUDOPOTENTIALS. Physical Review Letters 1982, 48, (20), 1425-1428.

13. Bylander, D. M.; Kleinman, L., OUTER-CORE ELECTRON AND VALENCE ELECTRON PSEUDOPOTENTIAL. Physical Review B 1984, 29, (4), 2274-2276.

14. Nose, S., A UNIFIED FORMULATION OF THE CONSTANT TEMPERATURE MOLECULAR-DYNAMICS METHODS. Journal of Chemical Physics 1984, 81, (1), 511-519.

15. Hoover, W. G., CANONICAL DYNAMICS - EQUILIBRIUM PHASE-SPACE DISTRIBUTIONS. Physical Review A 1985, 31, (3), 1695-1697.

16. Blochl, P. E.; Parrinello, M., ADIABATICITY IN 1ST-PRINCIPLES MOLECULAR-DYNAMICS. Physical Review B 1992, 45, (16), 9413-9416. 